\title{
MS05-P02 | Structural Studies Of TyPe II TNF-A From Orange-SPOTted Grouper, EPINEPHELUS COIOIDES
}

Lin, Shih-Ming (Department of Biotechnology and Bioindustry Sciences, National Cheng Kung University, Tainan City, TWN); Lin, John Han-You (Institute of Veterinary Medicine, National Taiwan University, Taipei, TWN); Kuo, Wan-Ching (Department of Biotechnology and Bioindustry Sciences, National Cheng Kung University, Tainan city, TWN); Yu, Ming (Department of Biotechnology and Bioindustry Sciences, National Cheng Kung University, Tainan city, TWN)

TNF- $\alpha$ is one of the most important cytokines regulating the immune system in vertebrates. It is mainly secreted by macrophage to induce multiple immune responses of innate immunity. So far, a single type of TNF- $\alpha$ is found in the mammalian system. However, it is known that two types of TNF- $\alpha$ exist in teleost fish. Revealing the structural and functional variety between teleost and mammalian TNF- $\alpha$ would be important to understand the immunoregulation in the vertebrates. Here, we present the crystal structure of type II TNF- $\alpha$ from Orange-spotted Grouper, Epinephelus coioides at $3.6 \AA ̊$ resolution. The EcTNF- $\alpha 2$ structure showed a typical jelly-roll fold with eight beta strands and formed a homotrimer complex like the mammalian TNF- $\alpha$ proteins. Superposition of each protomer showed the r.m.s.d. is small than $2 \AA$, indicating that the trimeric conformation does not have the orientational specificity. The interacting surfaces between protomers mainly consisted of hydrophobic residues and several highly conserved residues contribute to maintaining the robust trimeric conformation. The receptorinteracting regions of EcTNF- $\alpha 2$ are composed of several acidic residues, which means the electrostatic interaction is the major force to recognize the TNF- $\alpha$ receptor. Compare to the human TNF- $\alpha, E c T N F-\alpha 2$ showed a highly structural homology but the receptor binding sites are relatively different. This study provides evidence revealing the functional and structural homology in the immune system of vertebrates. Further studies on the type I teleost TNF- $\alpha$ will be conducted to reveal the differences between the two types of TNF- $\alpha$ proteins. 